\title{
VON UGARIT NACH QUMRAN
}

B E I TR ÄGE ZUR

ALTTESTAMENTLICHEN UND ALTORIENTALISCHEN

FOR S CHUNG

\section{OTTO EISSFELDT}

ZUM 1. SEPTEMBER 1957 DARGEBRACHT VON FREUNDEN UND SCHULERN,

HERAUSGEGEBEN IN ZUSAMMENARBEIT MIT

W. F. ALBRIGHT, W. BAUMGARTNER, J. LINDBLOM,

J. PEDERSEN und H. H. ROWLEY

VON

JOHANNES HEMPEL UND LEONHARD ROST

ZWEITE UNVERÄNDERTE AUFLAGE

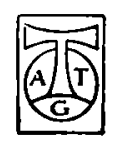

I96r

VERLA G ALFRED TÖPELMAN N B ERLIN 
BEIHEFTE ZUR ZEITSCIHRIFT FUR DIE

ALTTESTAMFNTIICHE WISSENSCHAFT 7 Gleichzeitig mit der ersten Auflage ist eine gebundene Ausgabe mit der Tabula
gratulatoria der Hallenser Fakultät, der Liste der Gratulanten und einem Bericht
über Vorgeschichte und Uberreichung der Festschrift erschienen

Alle Rechte, einschl. der Rechte der Herstellung von Photokopien und Mikrofilmen von der Verlagshandlung vorbehalten

Printed in Germany

Satz: Walter de Gruyter \& Co., Berlin W 30 